\title{
CDISC SDTM Tumor Response Result Terminology
}

National Cancer Institute

\section{Source}

National Cancer Institute. CDISC SDTM Tumor Response Result Terminology. NCI

Thesaurus. Code $C 96785$.

Terminology associated with the tumor response result codelist of the Clinical Data

Interchange Standards Consortium (CDISC) Study Data Tabulation Model (SDT M). 